\title{
Erratum: Seasonal Occurrence of Cownose Rays (Rhinoptera bonasus) in North Carolina's Estuarine and Coastal Waters
}

\author{
M. April Goodman • Paul B. Conn • Eric Fitzpatrick
}

Published online: 2 December 2010

(C) Coastal and Estuarine Research Federation 2010

Erratum to: Estuaries and Coasts

DOI 10.1007/s12237-010-9355-5

Eric Fitzpatrick's complete affiliations are as follows:

North Carolina Division of Marine Fisheries,

943 Washington Square Mall,

Washington, NC 27889, USA

Present address:

National Marine Fisheries Service,

Southeast Fisheries Science Center, Beaufort Laboratory,

101 Pivers Island Road,

Beaufort, NC 28516, USA

The online version of the original article can be found at http://dx.doi. org/10.1007/s12237-010-9355-5.

M. A. Goodman $(\bowtie) \cdot$ P. B. Conn

National Marine Fisheries Service,

Southeast Fisheries Science Center, Beaufort Laboratory,

101 Pivers Island Road,

Beaufort, NC 28516, USA

e-mail: April.Goodman@noaa.gov

E. Fitzpatrick

North Carolina Division of Marine Fisheries,

943 Washington Square Mall,

Washington, NC 27889, USA

Present Address:

E. Fitzpatrick

National Marine Fisheries Service,

Southeast Fisheries Science Center, Beaufort Laboratory,

101 Pivers Island Road,

Beaufort, NC 28516, USA 